\title{
Ultrasound-guided percutaneous needle biopsy skill for peripheral lung lesions and complications prevention
}

\author{
Hongxia Zhang", Yang Guang", Wen He, Linggang Cheng, Tengfei Yu, Yu Tang, Haiman Song, Xin Liu, \\ Yukang Zhang \\ Department of Ultrasound, Beijing Tiantan Hospital, Capital Medical University, Beijing, China \\ Contributions: (I) Conception and design: H Zhang, Y Guang; (II) Administrative support: W He; (III) Provision of study materials or patients: \\ L Cheng, Y Tang, Y Zhang; (IV) Collection and assembly of data: H Zhang; (V) Data analysis and interpretation: T Yu, H Song, X Liu; (VI) \\ Manuscript writing: All authors; (VII) Final approval of manuscript: All authors. \\ \#These authors contributed equally to this work as co-first authors. \\ Correspondence to: Wen He, MD. No. 119 West Road of South 4th Ring Road, Fengtai District, Beijing, China. Email: ttyyus@sina.com.
}

Background: To investigate puncture skills and complications prevention in ultrasound-guided percutaneous needle biopsy for peripheral lung lesions.

Methods: Ninety-two peripheral lung lesions in 92 patients, detected via computed tomography (CT) and also visible on ultrasound, were retrospectively analyzed. All patients underwent percutaneous peripheral lung lesion needle biopsy under traditional ultrasound or contrast enhanced ultrasound (CEUS) guidance paying attention to avoiding necrotic areas and large blood vessels. All the specimens were examined histopathologically. Preprocedure all 92 lesions were performed by traditional ultrasonography to evaluate the size, the echogenecity, liquefaction areas and blood flow on color Doppler imaging, some of which were performed by CEUS for evaluating non-enhanced necrosis areas, contrast agent arrival time (AT) and characteristics of blood perfusion.

Results: The histopathologic results of all 92 lesions were as follows: 67 malignant tumors (including 28 adenocarcinomas, 19 squamous cell carcinomas, 6 bronchoalveolar carcinomas, 5 small cell carcinomas, 5 metastatic cancers, 3 poorly differentiated cancers and 1 malignant mesothelioma), 20 benign lesions (including 9 pneumonia, 6 inflammatory pseudotumors and 5 tuberculomas), 5 undetermined lesions. Of 52 lesions by CEUS guidance, 7 lesions showed enhancement in the pulmonary arterial-phase (including 6 pneumonia and 1 malignant tumors), 45 lesions showed enhancement in the bronchial artery phase (including 37 malignant tumors, 3 inflammatory pseudotumors, 4 tuberculomas and 1 undetermined lesion). According to needle insertion angle along linear path, a total of 92 lesions were divided into two groups, 49 lesions at an angle of $70^{\circ}-80^{\circ}$ needle insertion and 43 lesions at an angle of $80^{\circ}-90^{\circ}$ needle insertion. In the study, linear and non-linear two puncture paths were used, we first tried to puncture along linear path in all lesions, if an attempt to insert into the lesions failed due to be blocked by the ribs and then changed to puncture along non-linear path instead. The success rate of biopsy procedure along linear puncture was significantly higher at an angle of $80^{\circ}-90^{\circ}$ group $(93.0 \%$ vs. $20.4 \%, \mathrm{P}<0.01)$, and the adoption rate of nonlinear path biopsy for solving the puncture needle blocked by the ribs was significantly higher at angle of $70^{\circ}-80^{\circ}$ group ( $79.6 \%$ vs. $7.0 \%, \mathrm{P}<0.01$ ). Of 52 lesions by CEUS guidance, 27 (51.9\%) showed non enhanced necrosis areas on CEUS, only 5 showed liquefaction necrosis areas on gray-scale ultrasound. Of 40 lesions by traditional ultrasound guidance, 4 showed necrosis areas on gray-scale ultrasound. There were no significant differences in lesion size, the average number of biopsy attempts and complication rates between CEUS guidance group and traditional ultrasound guidance group $(\mathrm{P}>0.05)$, the pathological confirmation rate in CEUS guidance group was higher than that in traditional ultrasound guidance group, but without significant difference (98.1\% vs. 90.0\%, P>0.05). Of all 92 cases, 3 cases (3.3\%) had mild pneumothorax and 4 cases $(4.3 \%)$ had hemoptysis.

Conclusions: In ultrasound-guided needle biopsy for peripheral lung lesions, using a combination of linear and non-linear puncture techniques and keeping away from necrotic areas and large blood vessels, may help to increase the success rate and reduce the incidence of complications further. 
Keywords: Lung; ultrasound; contrast-enhanced ultrasound; puncture

Submitted Sep 10, 2019. Accepted for publication Jun 02, 2020.

doi: $10.21037 /$ jtd-2019-abc-03

View this article at: http://dx.doi.org/10.21037/jtd-2019-abc-03

\section{Introduction}

With the wide application of high-resolution CT and the established role of low-dose helical computed tomography (CT) screening for lung cancer, pulmonary lesions are increasingly detected $(1,2)$. Image-guided biopsy is still required for lesions that cannot be determined by CT or malignant lesions diagnosed by CT need to be clarified histopathologically or genetic types before radiotherapy or chemotherapy (3-5). Percutaneous biopsy under CT guidance is often used for peripheral lung lesions nearby or adjacent to the chest wall, and percutaneous biopsy for peripheral lung lesions adjacent to the chest wall can be performed under both CT and ultrasound guidance. Compared with CT-guided biopsy, ultrasound-guided biopsy for peripheral lung lesions was simple, safe, realtime visualization, fewer complications, lower cost and no radiation exposure, the diagnosis rate similar to that of CT (5-8). The defect of ultrasound guidance is easy to be affected by aerated lung tissue, narrow scope of application (only suitable for peripheral lung lesions adjacent to the chest wall), in addition, mastering ultrasound-guided puncture biopsy requires a long training period, which may be the reason why CT guidance is currently preferred more than ultrasound guidance in lung lesions puncture biopsy in many countries $(9,10)$. In China, ultrasound-guided biopsy is usually preferred for peripheral lung lesions adjacent to the chest walls, while CT-guided puncture biopsy is mainly used for the pulmonary lesions that cannot be displayed by ultrasound. So far, few studies focused on the technical aspects of ultrasound-guided percutaneous peripheral lung lesions biopsy (11). This study aims to investigate the puncture skill of peripheral lung lesion biopsy under ultrasound guidance, so as to improve the success rate, reduce the incidence of complications and promote its clinical application.

\section{Methods}

\section{Patients}

Ninety-two peripheral pulmonary lesions in 92 patients, detected via CT and also visible on ultrasound, were retrospectively analyzed. CT could not determine benign or malignant in 53 cases, the histopathological type of malignant lesions needed to be identified before chemotherapy or radiotherapy in 39 cases. The study population included 56 males and 36 females with a mean age of $66.4 \pm 12.6$ years (range, $52-82$ years). Ninety-two peripheral lung lesions were a mean diameter of $3.2 \pm 1.1 \mathrm{~cm}$ (range, 1.2-9.2 cm). The study was conducted in accordance with the Declaration of Helsinki (as revised in 2013). This study was approved by the Ethics Committee of Beijing Tiantan Hospital (KY 2019-015-02), and written informed consents were obtained from all patients prior to contrast enhanced ultrasound (CEUS) and ultrasound-guided percutaneous needle biopsy.

\section{Equipment}

The GE Logiq E9 color Doppler ultrasound system equipped with a 1-5 $\mathrm{MHz}$ convex array probe, 2-7 MHz small convex array probe, and a probe-guiding puncture device was used. Bard automatic ejection biopsy gun and Bard disposable biopsy needle (16G, 18G) were used. A second-generation ultrasound contrast agent, SonoVue (Bracco Imaging, Milan, Italy) was used. Each time, $2.4 \mathrm{~mL}$ of the contrast agent suspension was injected into the cubital vein, followed by injection of $5.0 \mathrm{~mL}$ of saline solution as a flush. Imaging software with a low mechanical index was used to analyze the images.

\section{Biopsy methods}

Preprocedure ultrasonography examination was performed in all 92 lesions in 92 patients for the lesions' size, echogenecity, liquefaction areas and blood flow on color Doppler imaging, of whom CEUS was performed in 52 lesions in 52 consecutive patients for observing nonenhanced necrosis areas, contrast agent arrival time (AT) and characteristics of blood perfusion in the lesions, which aimed to investigate the localization sampling by CEUS on the histopathologic results and the characteristics of blood 

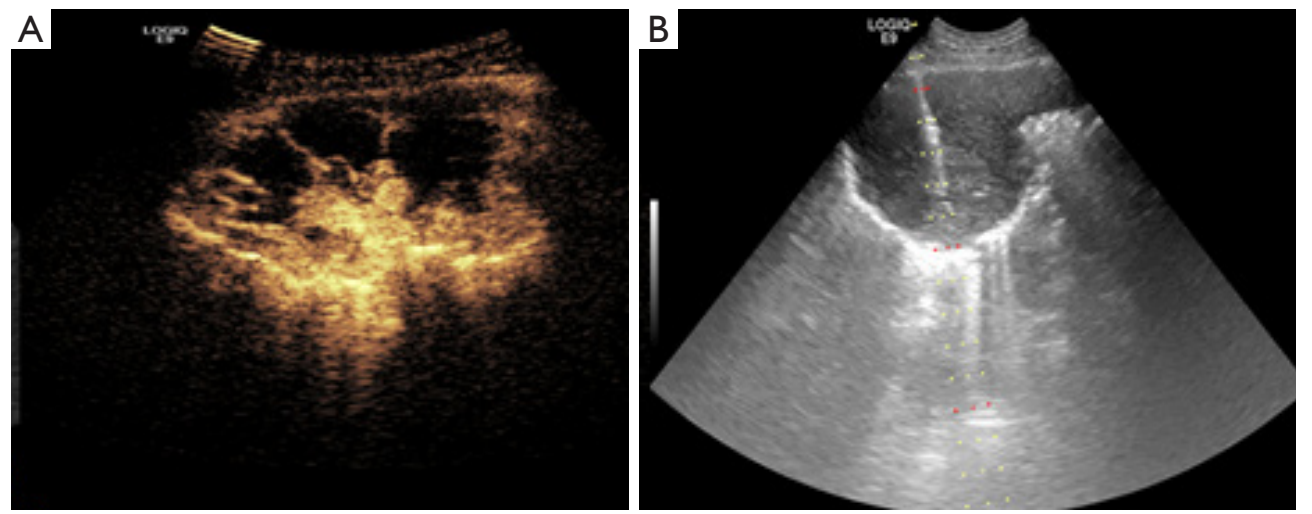

Figure 1 The lesion was punctured avoiding non enhanced necrosis area under guidance of contrast-enhanced ultrasound (CEUS). (A) CEUS displayed irregular non enhanced necrosis areas in the lesion pre-procedure; (B) needle puncture was performed under gray scale ultrasound-guidance avoiding non enhanced necrosis area by CEUS.
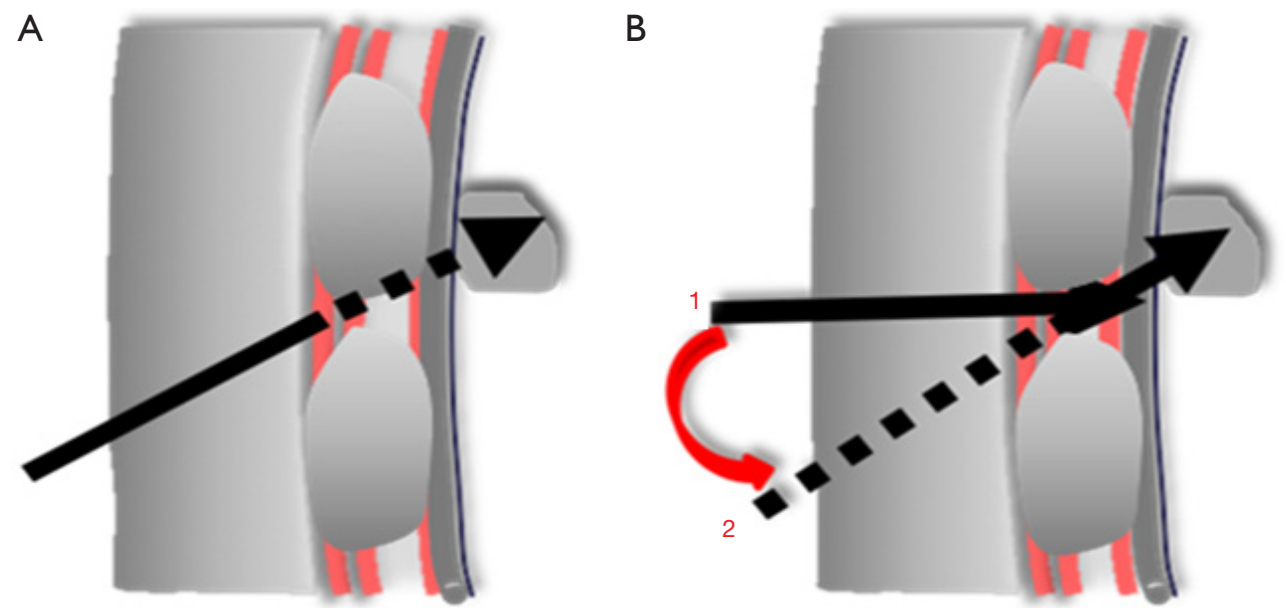

Figure 2 Schematic diagram of needle biopsy for peripheral pulmonary lesions behind ribs along linear path and non-linear path. (A) Along linear path: Biopsy failed due to the needle hindered by the rib; (B) Along non-linear path: Biopsy completed avoiding the rib successfully through deflecting the needle from position 1 to position 2 (the solid line representing the actual track of the needle, the dotted line representing the position of the needle after deflection, the arrow representing the position of the needle tip).

perfusion of different lung lesions.

The arterial phase was determined by comparing contrast agent AT in the peripheral lung lesion with that in adjacent lung tissue using "real-time comparative observation method" (12). All patients underwent percutaneous peripheral lung lesion biopsy under traditional ultrasound or CEUS guidance paying attention to avoiding necrotic areas and large blood vessels (Figure 1). All the specimens were examined pathologically. None of the patients had contraindications for CEUS and the biopsy, and signed informed consent was obtained from each patient or family members before the CEUS and biopsy procedures.

In the study, linear and non-linear two puncture paths were employed, we first tried to puncture along traditional linear path in all lesions, if an attempt to puncture the lesions directly failed due to be blocked by the ribs and then changed to puncture along non-linear path instead (Figure 2). (I) The traditional linear path: needle insertion point on skin was at the center of intercostal space with the nearest distance to the lesion and the lesion displayed clearly. A needle tract was designed to pass through the needle insertion point and the target area of the lesion, 
being coincidence with built-in puncture guide line. Then the puncture needle was put into the needle groove of the guide device and the whole biopsy process was guided by real-time ultrasound; (II) non-linear path: if an attempt to puncture the lesion directly along linear path failed due to be blocked by the rib along linear path and then changed to puncture along non-linear path instead. The needle insertion point on skin remained unchanged, the puncture needle subverticaly descended through the intercostal space down to the inner intercostal muscle layers, then tilting the probe with puncture needle properly to make the direction of the needle in line with the preset puncture guide line across the target lesion, then puncture needles were penetrated into the lesion along the guide line under realtime guidance of ultrasound and specimens were obtained.

\section{Statistical methods}

SPSS version 20.0 software was used for statistical analysis. Fisher's test or the chi-squared test was used to compare qualitative data. The two-sample $t$-test was used to compare the number of punctures between the two groups. $\mathrm{P}<0.05$ represented a statistically significant difference.

\section{Results}

The histopathologic results of all 92 lesions were as follows: 67 malignant tumors (including 28 adenocarcinomas, 19 squamous cell carcinomas, 6 bronchoalveolar carcinomas, 5 small cell carcinomas, 5 metastatic cancers, 3 poorly differentiated cancers and 1 malignant mesothelioma); 20 benign lesions (including 9 pneumonia, 6 inflammatory pseudotumors and 5 tuberculomas); 5 undetermined lesions. Of 52 lesions by CEUS, 7 lesions showed enhancement in the pulmonary arterial-phase (including 6 pneumonia and 1 malignant tumor), 45 lesions showed enhancement in the bronchial artery phase (including 37 malignant tumors, 4 inflammatory pseudotumors, 3 tuberculomas and 1 undetermined lesion).

Total of 92 peripheral lung lesions were note with mean diameter of $3.2 \pm 1.1 \mathrm{~cm}$ (range, 1.2-9.2 cm), including 39 small lesions with a diameter of 1-2 cm and 53 large lesions with a diameter greater than or equal to $2 \mathrm{~cm}$. All 92 lesions had either uniform or inhomogeneous hypoechogenicity on gray-scale ultrasound, 79 lesions were hypovascular and 13 lesions were hypervascular on Doppler imaging. Fiftytwo patients were performed percutaneous biopsy by CEUS guidance and 40 patients were performed by traditional ultrasound guidance. Of 52 lesions by CEUS guidance, 27 (51.9\%) showed non enhanced necrosis areas on CEUS and only 5 of them showed liquefaction necrosis areas on gray-scale ultrasound, a large narrow base lesion sized $3.5 \mathrm{~cm}$ for high risk of pneumothorax was ignored on grayscale ultrasound and discovered by CEUS preprocedure, the other 51 lesions displayed with wide base on grayscale ultrasound and CEUS (Figures 3,4). Of 40 lesions by traditional ultrasound guidance, 4 showed liquefaction necrosis areas on gray-scale ultrasound.

In 82 cases $18 \mathrm{G}$ biopsy needle was used and in 10 cases $16 \mathrm{G}$ biopsy needle was used. The average numbers of puncture was 3.0 (range, 1-5). Mild pneumothorax occurred in $3(3.3 \%)$ cases: 2 small lesions with a diameter of 1-2 cm and 1 large lesion with a diameter of $3.5 \mathrm{~cm}$. Hemoptysis occurred in 4 (4.3\%) cases, massive hemoptysis in 1 case during procedure and bloody sputum in 3 cases postprocedure. The average number of biopsy attempts by traditional ultrasound and CEUS guidance was 3.1 and 3.0, respectively. There were no significant differences in lesion size, the average number of biopsy attempts and complication rates between two groups $(\mathrm{P}>0.05)$. The pathological confirmation rate in CEUS guidance group was higher than that in traditional ultrasound group, but without significant difference ( $98.1 \%$ vs. $90.0 \%, \mathrm{P}>0.05$ ) (Table 1).

According to needle insertion angle along linear path, a total of 92 lesions were divided into two groups, 49 lesions at an angle of $70^{\circ}-80^{\circ}$ needle insertion and 43 lesions at an angle of $80^{\circ}-90^{\circ}$ needle insertion, the success rate of biopsy procedure along linear puncture was significantly higher at an angle of $80^{\circ}-90^{\circ}$ group $(93.0 \%$ vs. $20.4 \%, \mathrm{P}<0.01)$, and the adoption rate of non-linear path biopsy for solving the puncture needle blocked by the ribs was significantly higher at angle of $70^{\circ}-80^{\circ}$ group ( $79.6 \%$ vs. $7.0 \%, \mathrm{P}<0.01$ ) (Table 2). The over all adoption rate of non-linear path biopsy is $45.7 \%$ (42/92).

\section{Discussion}

The needle insertion angle is mainly dependent on the position of the lesion. According to needle insertion angle along linear path, lesions were divided into $70^{\circ} \sim 80^{\circ}$ angle group and $80^{\circ}-90^{\circ}$ angle group in our study, the success rate of biopsy procedure along linear puncture was significantly higher at angle of $80^{\circ}-90^{\circ}$ group $(93.0 \%$ vs. $20.4 \%, \mathrm{P}<0.01)$, and the adoption rate of non-linear path biopsy for solving the puncture needle blocked by the ribs was significantly higher at angle of $70^{\circ}-80^{\circ}$ group $(93.0 \%$ vs. $20.4 \%, \mathrm{P}<0.01)$. 

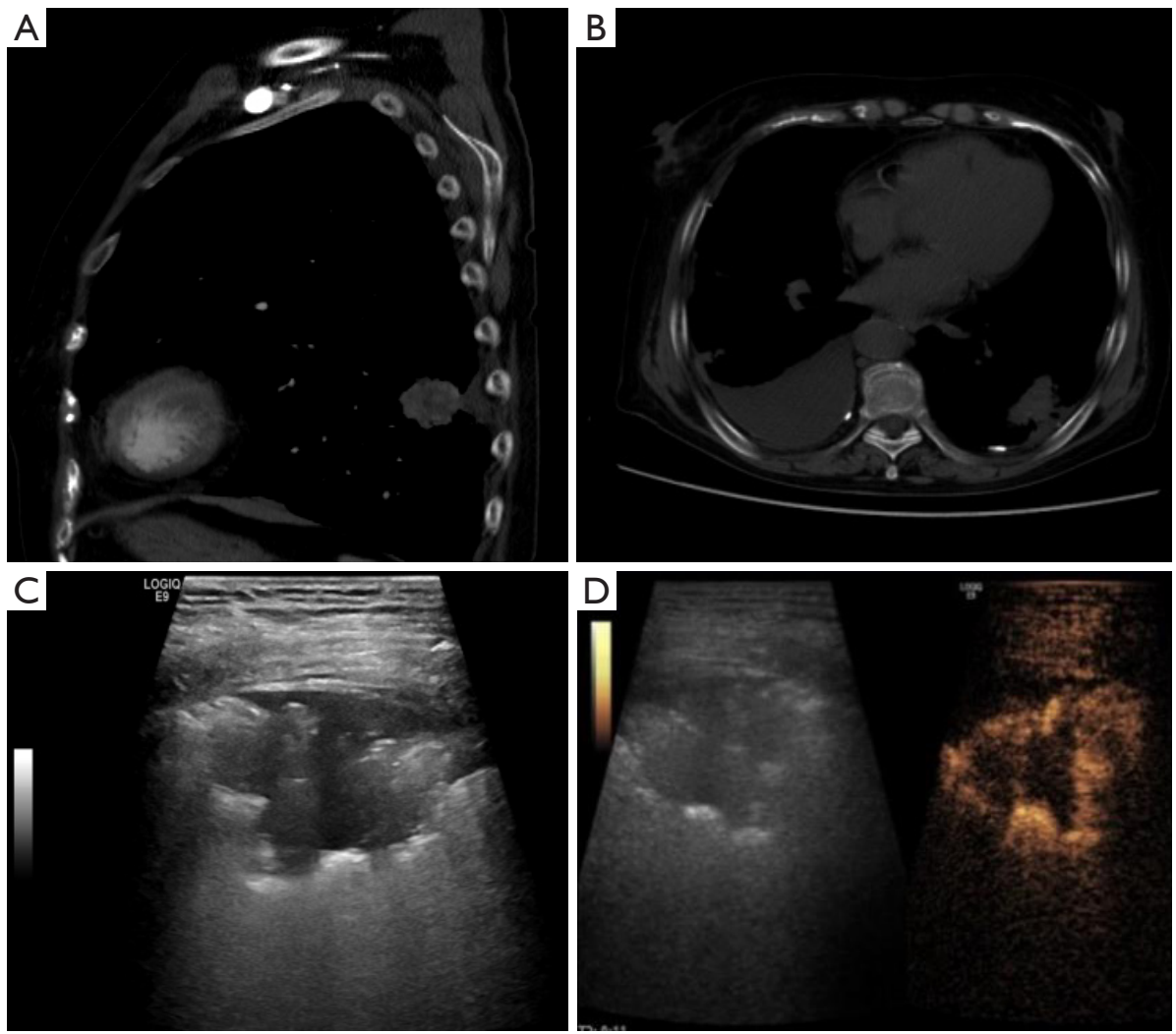

Figure 3 A narrow-based periphery lung lesion (cancer nodule) on computed tomography (CT), gray-scale ultrasound and contrast enhanced ultrasound (CEUS). (A) CT imaging scan in sagittal view; (B) CT imaging scan in transverse view; (C) being affected by gas interference, the lesion displays unclear edge hypoechoic area on gray-scale ultrasound; (D) the lesion displays a circular high enhancement around it in early phase on CEUS.
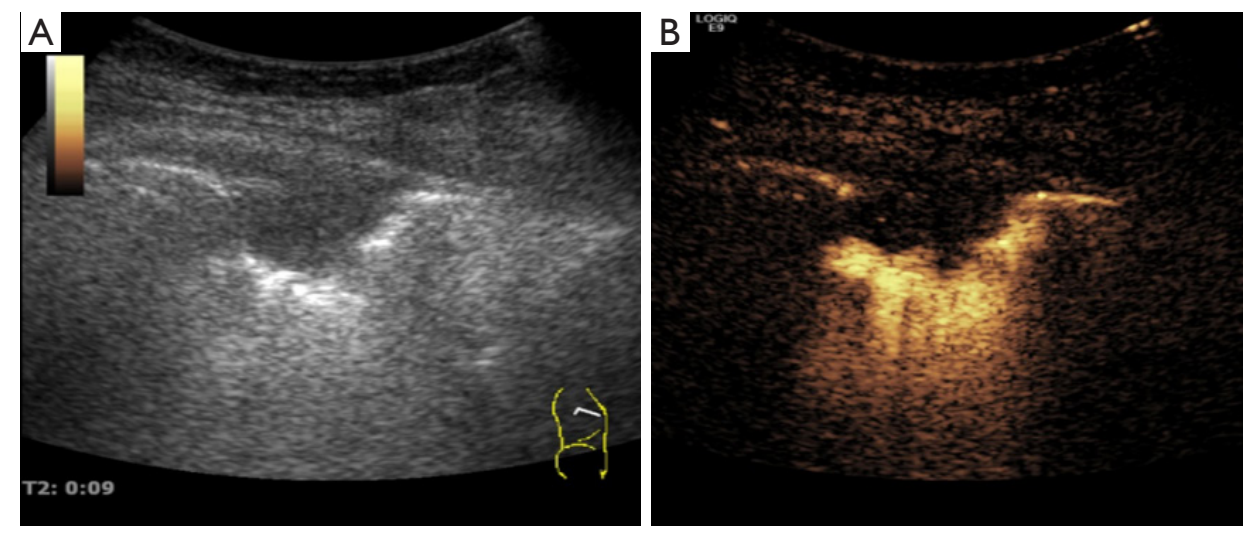

Figure 4 A wide-based peripheral lung lesion (tuberculosis nodule) on gray-scale ultrasound and contrast enhanced ultrasound (CEUS). (A) The lesion shows hypoechoic area with clear boundary on gray-scale ultrasound; (B) the lesion displays a semi-circular high enhancement around it in early phase on CEUS. 
Table 1 Comparison between traditional ultrasound guided biopsy and CEUS guided biopsy with histopathologic results and complications

\begin{tabular}{|c|c|c|c|}
\hline Indicator & Traditional ultrasound guidance $(n=40)$ & CEUS guidance $(n=52)$ & $P$ value \\
\hline$<2$ & 17 & 22 & \\
\hline$>2$ & 23 & 30 & \\
\hline Histopathologic results & & & $>0.05$ \\
\hline unconfirmed & 4 & 1 & \\
\hline Complications & & & $>0.05$ \\
\hline Pneumothorax & 2 & 1 & \\
\hline Hemoptysis & 2 & 2 & \\
\hline
\end{tabular}

CEUS, contrast enhanced ultrasound.

Table 2 Relationship between needle insertion angle along linear path and the actual puncture path

\begin{tabular}{lcc}
\hline \multirow{2}{*}{ Needle insertion angle along linear path } & \multicolumn{2}{c}{ The actual puncture path (\%) } \\
\cline { 2 - 3 } & Non-linear & Linear path \\
\hline $80^{\circ}-90^{\circ}(\mathrm{n}=43)$ & $3(7.0)$ & $40(93.0)$ \\
$70^{\circ}-80^{\circ}(\mathrm{n}=49)$ & $39(79.6)$ & $10(20.4)$ \\
\hline
\end{tabular}

In the study, the problem of puncture needles blocked by the ribs along linear path biopsy was completely solved using non-linear path biopsy, which was attributed to the fact that the lesions at $80^{\circ}-90^{\circ}$ subvertical angle needle insertion usually located in the intercostal space and biopsy along linear path had a high successful rate, while lesions at an angle of $70^{\circ}-80^{\circ}$ needle insertion usually located behind the ribs and biopsy along non-linear path was appropriate. Therefore, in percutaneous ultrasound-guided biopsy for peripheral lung lesions, the angle of needle insertion may serve as a reminder of the appropriate puncture path.

In percutaneous ultrasound-guided biopsy for peripheral lung lesions, the problem of puncture needles blocked by the ribs is a frequent occurrence, especially for lesions located behind the ribs. Ultrasound image has poor resolution for needle tips and lesions and chest wall compared with CT image influenced by the aerated lung, so, regardless of anatomical features of peripheral lung lesions and the chest wall, blindly trying can easily lead to biopsy failure and increase the incidence of complications and is not advisable In the study, the needle was firstly punctured along traditional linear path in all lesions, if the needle tip happened to be blocked by the rib and then changed to puncture along non-linear path instead. In percutaneous ultrasound-guided biopsy for peripheral lung lesions, puncture biopsy along linear path by realtime guidance of ultrasound is most commonly performed, simple and convenient, preferable for lesions located in the intercostal space, but prone to failure for lesions located behind the ribs, we solved this problem well using "nonlinear path" puncture technique. The over all adoption rate of non-linear path biopsy is $45.7 \%$ (42/92) in the paper. Obviously, "linear" and "non-linear" puncture path are suited for lesions located in the intercostal space and behind the ribs respectively, which are equally important skills in ultrasound-guided needle biopsy for peripheral lung lesions.

The most common complication of ultrasoundguided percutaneous biopsy for peripheral lung lesions is pneumothorax, the incidence of pneumothorax is $1-6 \%$ (13-15). In the paper, the incidence of pneumothorax in this study was $3.3 \%$ and all were mild pneumothorax without thoracic intubation and drainage, which was similar to relevant literature reports by ultrasound guidance and far lower than the incidence of CT pneumothorax at 20.5\% (5). Pneumothorax occurs easily in small lesions with a diameter of $1-2 \mathrm{~cm}$, especially those around $1 \mathrm{~cm}$ in diameter, which is 
mainly related to the deviation of the guidance, the patient's respiratory movement and the presence of gas reverberation artifacts. Priolal reported that the risk of pneumothorax in lesions smaller than $2 \mathrm{~cm}$ may be 17 -fold higher than in larger lesions (16). In the paper, 3 cases had pneumothorax, 2 cases with small lesion size of $1-2 \mathrm{~cm}$ and 1 case with a large lesion size of $3.5 \mathrm{~cm}$, the latter patient's pneumothorax was due to her narrow-based lesion less than $1 \mathrm{~cm}$ like a pedicle, it was first ignored on gray-scale ultrasound because of lack of typical features and discovered by CEUS later. In early phase on CEUS, the narrow-based lesion displayed circular high enhancement around it and the wide-based lesion displayed semi-circular high enhancement around it, which was due to retaining normal the pulmonary arteries perfusion in the air-conditioning lung tissue around the lesion. Pneumothorax complication in this case was almost inevitable, fortunately we seized the only opportunity for biopsy and obtained "non-mucinous alveolar carcinoma" confirmed pathological findings depending on one slice of lesion's tissue sample. So, in ultrasound-guided biopsy for peripheral lung lesions, we should pay attention not only to the size of the lesion but also to the base width of the lesion preprocedure $(10,11)$, CT imaging for lung lesions being more accurate and reliable than US, it is very important for understanding the characteristics of lesions by CT imaging in ultrasound-guided biopsy for peripheral lung lesions. Except for the lesion's size and the base width, breath control is also very important to prevent pneumothorax, all patients should be trained to hold breath preprocedure. In ultrasound-guided biopsy for peripheral lung lesions, once pneumothorax occurs, the lesion cannot be displayed and biopsied repeatedly, "one shot" biopsy should be prepared for patients with high risks of pneumothorax.

Another common complication in percutaneous biopsy for peripheral lung lesions is hemorrhage, being characterized by a small amount of minor bleeding in most cases, occasionally severe complications such as hemoptysis, hemothorax or intrapulmonary hematoma may occur $(17,18)$. From the perspective of precaution, the thickness of the biopsy needle should be paid more attention. At present, 18G biopsy needles with moderate thickness are typically used, a few scholars attempted to use $16 \mathrm{G}$ or $14 \mathrm{G}$ biopsy needles for peripheral lung lesion and thought there was no significant increase in the incidence of complications but the greater tissue yield can reduce the number of punctures (19-21). In the study, 16G biopsy needles were used in 10 patients, and one patient showed hemorrhage in the sputum. Statistical analysis was not performed because the number of cases was small and case selection was not random. The incidence of hemoptysis in this study was $4.3 \%$, which was similar to the literature reports (13-15). To note that, in our study 1 case was complicated by serious hemoptysis during the procedure due to only a puncture by $18 \mathrm{G}$ needle (the histopathological findings "lung adenocarcinoma"), who had a large lesion sized $9.2 \mathrm{~cm}$ in diameter and high vascularity, cough and bloody sputum preprocedure, bleeding gradually stopped but obstructive atelectasis occurred in affected lung lobe post procedure. In all, hemorrhage complication is mild and rarely happened in peripheral lung lesions biopsy ultrasound-guided, but massive hemorrhage complication should be paid more attention in large lesions due to lesions with abundant blood supply and invasion of deep large vessels. It is helpful for preventing hemorrhage complications by using thick needles with caution, restricting puncture attempts, puncturing at relatively shallow areas with no large blood vessels.

Priolal reported that the diagnostic accuracy rate of lesions $>5 \mathrm{~cm}$ in size was $78 \%$, and the diagnostic accuracy rate of lesions $<5 \mathrm{~cm}$ in size was $87 \%$, which was related to the high incidence of liquefaction necrosis (16). CEUS is more sensitive and reliable than traditional ultrasound in identifying focal necrosis of lesions, needle biopsy of peripheral lung lesions under CEUS guidance is receiving increasing attention in recent years (12,22-27). In the study, preprocedure CEUS was performed in 52 lesions in 52 consecutive patients to evaluate non-enhanced focal necrosis and determine the lesions' sampling part in order to improve histopathologic confirmation rate of puncture biopsy, 27 (51.9\%) showed no enhanced focal necrosis, and among them only 5 showed liquefaction necrosis on grayscale ultrasound. The pathological confirmation rate of puncture biopsy under CEUS guidance was higher than that under traditional ultrasound guidance, but no significant difference was observed, which perhaps due to multiple puncture attempts and small numbers. Beyond above, CEUS can also be used for the preliminary qualitative evaluation of peripheral lung lesions. Previous studies have shown that blood supply to pulmonary malignant tumors originates mainly from the bronchial arteries and manifests as delayed enhancement on CEUS, pneumonia, atelectasis and pulmonary consolidation, on the other hand, exhibit early enhancement due to retained normal blood perfusion $(12,22-27)$, our results conform to that dealt with in the literature. In addition, we also found that tuberculous nodules and inflammatory granulomas chronic 
inflammatory benign lesions showed delayed enhancement similar to malignant tumors, of whom the similarity of blood perfusion patterns may be an important reason hard to distinguish from one another by various imaging techniques. With image technology progressing faster than ever, biopsy for histopathological examination guided by various images (including ultrasound) is still irreplaceable in lung lesions.

\section{Conclusions}

In ultrasound-guided needle biopsy for peripheral lung lesions, using a combination of linear and non-linear puncture techniques and keeping away from necrotic areas and large blood vessels, may help to increase the success rate and reduce the incidence of complications further.

\section{Acknowledgments}

Funding: None.

\section{Footnote}

Conflicts of Interest: All authors have completed the ICMJE uniform disclosure form (available at http://dx.doi. org/10.21037/jtd-2019-abc-03). The authors have no conflicts of interest to declare.

Ethical Statement: The authors are accountable for all aspects of the work in ensuring that questions related to the accuracy or integrity of any part of the work are appropriately investigated and resolved. The study was conducted in accordance with the Declaration of Helsinki (as revised in 2013). This study was approved by the Ethics Committee of Beijing Tiantan Hospital (KY 2019015-02), and written informed consents were obtained from all patients prior to CEUS and ultrasound-guided percutaneous needle biopsy.

Open Access Statement: This is an Open Access article distributed in accordance with the Creative Commons Attribution-NonCommercial-NoDerivs 4.0 International License (CC BY-NC-ND 4.0), which permits the noncommercial replication and distribution of the article with the strict proviso that no changes or edits are made and the original work is properly cited (including links to both the formal publication through the relevant DOI and the license). See: https://creativecommons.org/licenses/by-nc-nd/4.0/.

\section{References}

1. Lim W, Ridge CA, Nicholson AG, et al. The 8th lung cancer TNM classification and clinical staging system: review of the changes and clinical implications. Quant Imaging Med Surg 2018;8:709-18.

2. Barton $\mathrm{H}$, Shatti $\mathrm{D}$, Jones CA, et al. Review of radiological screening programmes for breast, lung and pancreatic malignancy. Quant Imaging Med Surg 2018;8:525-34.

3. Moyer VA. Screening for lung cancer: U.S. Preventive services task force recommendation statement. Ann Intern Med 2014;160:330-8.

4. Kauczor HU, Bonomo L, Gaga M, et al. Esr/ers white paper on lung cancer screening. Eur Respir J 2015;46:28-39.

5. Tekin AF, Turgut B, Öncü F. Should We Perform Transthoracic Trucut Lung Biopsies of Pleural-Based Lung Masses Under Ultrasound Guidance or Computed Tomography Guidance? Ultrasound Q 2020;36:49-53.

6. Christiansen IS, Clementsen PF, Bodtger U, et al. Transthoracic ultrasound-guided biopsy in the hands of chest physicians - a stepwise approach. Eur Clin Respir J 2019;6:1579632.

7. Fontalvo LF, Amaral JG, Temple M, et al. Percutaneous us-guided biopsies of peripheral pulmonary lesions in children. Pediatr Radiol 2006;36:491-7.

8. Diacon AH, Schuurmans MM, Theron J, et al. Safety and yield of ultrasound-assisted transthoracic biopsy performed by pulmonologists. Respiration 2004;71:519-22.

9. DiBardino DM, Yarmus LB, Semaan RW. Transthoracic needle biopsy of the lung. J Thorac Dis 2015;7:S304-16.

10. Yamamoto N, Watanabe T, Yamada K, et al. Efficacy and safety of ultrasound (US) guided percutaneous needle biopsy for peripheral lung or pleural lesion: comparison with computed tomography (CT) guided needle biopsy. J Thorac Dis 2019;11:936-43.

11. Jeon KN, Bae K, Park MJ, et al. Us-guided transthoracic biopsy of peripheral lung lesions: Pleural contact length influences diagnostic yield. Acta Radiol 2014;55:295-301.

12. Hong-Xia Z, Wen H, Ling-Gang C, et al. A new method for discriminating between bronchial and pulmonary arterial phases using contrast-enhanced ultrasound. Ultrasound Med Biol 2016;42:1441-9.

13. Deng CJ, Dai FQ, Qian K, et al. Clinical updates of approaches for biopsy of pulmonary lesions based on systematic review. BMC Pulm Med 2018;18:146.

14. Horeweg N, van Rosmalen J, Heuvelmans MA, et al. Lung cancer probability in patients with ct-detected 
pulmonary nodules: A prespecified analysis of data from the nelson trial of low-dose ct screening. Lancet Oncol 2014;15:1332-41.

15. Lee SM, Park CM, Lee KH, et al. C-arm cone-beam ctguided percutaneous transthoracic needle biopsy of lung nodules: Clinical experience in 1108 patients. Radiology 2014;271:291-300.

16. Priola AM, Priola SM, Cataldi A, et al. Accuracy of ctguided transthoracic needle biopsy of lung lesions: Factors affecting diagnostic yield. Radiol Med 2007;112:1142-59.

17. Heerink WJ, de Bock GH, de Jonge GJ, et al. Complication rates of CT-guided transthoracic lung biopsy: Meta-analysis. Eur Radiol 2017;27:138-48.

18. Boskovic T, Stanic J, Pena-Karan S, et al. Pneumothorax after transthoracic needle biopsy of lung lesions under CT guidance. J Thorac Dis 2014;6 Suppl 1:S99-107.

19. Geraghty PR, Kee ST, McFarlane G, et al. Ct-guided transthoracic needle aspiration biopsy of pulmonary nodules: Needle size and pneumothorax rate. Radiology 2003;229:475-81.

20. Schulze R, Seebacher G, Enderes B, et al. Complications in CT-guided, semi-automatic coaxial core biopsy of potentially malignant pulmonary lesions. Rofo 2015;187:697-702.

21. Ocak S, Duplaquet F, Jamart J, et al. Diagnostic accuracy

Cite this article as: Zhang $\mathrm{H}$, Guang $\mathrm{Y}, \mathrm{He} \mathrm{W}$, Cheng L, Yu T, Tang Y, Song H, Liu X, Zhang Y. Ultrasound-guided percutaneous needle biopsy skill for peripheral lung lesions and complications prevention. J Thorac Dis 2020;12(7):3697-3705. doi: 10.21037/jtd-2019-abc-03 and safety of CT-guided percutaneous transthoracic needle biopsies:14-gauge versus 22-gauge needles. J Vasc Interv Radiol 2016;27:674-81.

22. Sartori S, Postorivo S, Vece FD, et al. Contrast-enhanced ultrasonography in peripheral lung consolidations: What's its actual role? World J Radiol 2013;5:372-80.

23. Görg C. Transcutaneous contrast-enhanced sonography of pleural-based pulmonary lesions. Eur J Radiol 2007;64:213-21.

24. Caremani M, Benci A, Lapini L, et al. Contrast enhanced ultrasonography (CEUS) in peripheral lung lesions: A study of 60 cases. J Ultrasound 2008;11:89-96.

25. Cao BS, Liang YM, Li XL, et al. Contrast-enhanced sonography of juxtapleural pulmonary tuberculoma. J Ultrasound Med 2013;32:749-56.

26. Sperandeo M, Sperandeo G, Varriale A, et al. Contrastenhanced ultrasound (CEUS) for the study of peripheral lung lesions: A preliminary study. Ultrasound Med Biol 2006;32:1467-72.

27. Bai J, Yang W, Wang S, et al. Role of arrival time difference between lesions and lung tissue on contrastenhanced sonography in the differential diagnosis of subpleural pulmonary lesions. J Ultrasound Med 2016;35:1523-32. 